\title{
Influence of SBA- I 5-type ordered mesoporous silica on ibuprofen loading and releasing of electrospun polyacrylonitrile nanofibers
}

\begin{abstract}
Due to noticeable textural and structural properties of ordered mesoporous materials, they are potent to be used as drug delivery systems, catalysts, sensors, and filtration systems. The aim of this work is to study the influence of rod-like SBA-15 type ordered mesoporous silica on drug absorption of electrospun polyacrylonitrile (PAN) nanofibers as additives particles. Electrospun PAN and PAN-charged webs have been prepared by electrospinning of PAN/DMF solution and the dispersion of SBA-15 particles in the PAN/DMF solution, respectively. Ibuprofen that is highly used as nonsteroidal and anti-inflammatory medicine has been selected as drug model to evaluate the drug absorption of both electrospun PAN and electrospun PAN/SBA-15 webs with the aid of ultraviolet visible spectroscopy measurement.
\end{abstract}

Collected results demonstrate an increase in drug absorption of electrospun PAN nanofibers by the presence of SBA-15 type ordered mesoporous silica particles in the polymeric nanofibers structure.

Keywords: electrospinning, nanofibers, ibuprofen (IBU), SBA-15 type ordered mesoporous silica
Volume 5 Issue I - 2019

\author{
Elham Mohsenzadeh,' Nabyl Khenoussi,' \\ Amir Houshang Hekmati, ${ }^{2}$ Laurence \\ Schacher,' Dominique Adolphe,' A Sezai \\ Saraç, ${ }^{3}$ Magali Bonne, ${ }^{4}$ Jocelyne Brendlé, ${ }^{4}$ \\ Bénédicte Lebeau, ${ }^{4}$ Hale Canbaz Karakaș ${ }^{3}$ \\ 'Laboratoire de Physique et Mécanique Textiles, Université de \\ Haute Alsace (UHA), Université de Strasbourg, France \\ ${ }^{2}$ Department of Textile Engineering, Faculty of Engineering, \\ South Tehran Branch, Islamic Azad University, Iran \\ ${ }^{3}$ Department of Chemistry Polymer Science and Technology, \\ Faculty of Sciences and Letters, Istanbul Technical University, \\ Turkey \\ ${ }^{4}$ Univ de Haute Alsace (UHA), CNRS, IS2M UMR 736I, \\ Université de Strasbourg, France
}
Correspondence: Dr. Elham Mohsenzadeh, Teacher assistant, Laboratoire de Physique et Mécanique Textiles, EA 4365, Université de Haute Alsace (UHA), Mulhouse, Université de Strasbourg, France, Tel +33(0)328384858, Email elham.mohsenzde@yahoo.com

Received: December 20, 2018| Published: January 14, 2019

\section{Introduction}

Electrospinning technique is well known as an efficient and simple method to fabricate fibers with very small diameters in the range of 10 to 1000 nanometers, and to produce nanofibers filled with various particles from both natural, synthetic, and blend polymers. ${ }^{1-3}$ The large surface area to volume ratio and high porosity of the electrospun webs make them good candidates for many different applications in various fields. Nowadays, this method is commonly used in many areas such as materials engineering, filtration, catalysis, Li-batteries, sensors, pharmaceutical, optical electronics, healthcare, chemistry, and biomedical. The electrospun webs could also be easily fabricated into various shapes such as membrane, tube, etc. ${ }^{4-6}$

Over the past decade many progresses have been done in pharmaceutical technology and particularly on drug efficient uses for patients. In order to reduce intensive medication many studies have focused on the drug delivery system development. The drug delivery system provides the ability to transfer the desired guest molecule to the targeted place and release it in a controlled manner. ${ }^{7-9}$ To achieve this goal a carrier that can load high amount of drug molecules and at the same time can control the drug release with a proper rate is needed. ${ }^{10-12}$ Nowadays, ordered mesoporous silica particles are more and more used as drug carriers because of their unique and interesting properties such as highly ordered structure, uniform pore size, large pore volume and high thermal stability. ${ }^{13-19}$ SBA-15 type ordered mesoporous silica is a two-dimensional, hexagonal mesoporous silica whose structure does not change by loading it with drugs. ${ }^{20,21}$ This structural stability makes SBA-15 particle to be a good candidate as a drug host. ${ }^{22-25}$

The high pore volume of SBA-15 type ordered mesoporous silica presents the possibility to load a large amount of drug and their large surface area helps them to have high potential for drug absorption. SBA-15 particle is covered with silanol groups with the possibility of modification on the inner surface of the existence pores and its external surface. Hydrogen bonding and the presence of silanol groups give this particle the ability to control the interaction with physiological fluids. ${ }^{26-28}$

In this study the surface to volume ratio of the electrospun nanofibers is increased by using the accessible mesoporous silica particles within the electrospun PAN nanofibers that makes them an appropriate substrate for many potential applications such as catalysis, sensors, separation, sorption, and drug delivery system. Therefore, it is expected that produced electrospun PAN webs charged by SBA-15 type ordered mesoporous silica demonstrate improved drug loading capacity compared to the pristine electrospun PAN nanofibers.

\section{Materials and methods}

Polyacrylonitrile (PAN) with the molecular weight $\mathrm{M}_{\mathrm{w}}=150,000 \mathrm{~g}$. $\mathrm{mol}^{-1}$ and ibuprofen with the molecular weight $\mathrm{M}_{\mathrm{w}}=206.28 \mathrm{~g} \cdot \mathrm{mol}^{-1}$ as a drug model have been supplied from Sigma-Aldrich (France). Pure N,N-dimethylformamide (DMF) (impurities $<152 \mathrm{ppm}$ ) has been purchased from Fisher Scientific (France). SBA-15 type ordered mesoporous silica particles (specific surface area $S_{B E T}=700 \mathrm{~m}^{2} \mathrm{~g}^{-1}$, 
average pore diameter $=7 \mathrm{~nm}$ ) has been synthesized according to the procedure reported in the previous work related to the electrospinning of PAN nanofibers incorporating with this kind of particles.

Single needle electrospinning machine that is consisted of syringe pump $(0.001-5 \mathrm{~mL} / \mathrm{h} \pm 0.001)$, high voltage power supply (0$30 \mathrm{KV} \pm 0.1)$, non-beveled needle $(0.7 \times 30 \mathrm{~mm} \times \mathrm{mm})$, and a Teflon collector charged by graphite have been used in this study.

\section{Producing electrospun PAN and PAN/SBA-I5 nanowebs}

PAN solution has been prepared by dissolving $12 \mathrm{wt} . \%$ of PAN polymer pellets into 88 wt.\% of dimethylformamide (DMF) by mechanical stirring of 24 hours in order to obtain a homogenous solution. Electrospinning process has been performed for 14 hours to obtain $400 \mathrm{mg}$ of electrospun PAN nanofibers. Table 1 presents the optimal electrospinning parameters and conditions to produce homogenous PAN nanofibers.

Table I Electrospinning parameters - electrospun PAN nanofibers

\begin{tabular}{ll}
\hline Electrospinning parameters & Values \\
\hline Applied voltage $(\mathrm{kV})$ & 14.5 \\
Needle to collector distance $(\mathrm{cm})$ & 20 \\
Needle gauge $(\mathrm{mm} \times \mathrm{mm})$ & $0.7 \times 30$ \\
Feed rate $(\mathrm{mL} / \mathrm{h})$ & 0.212 \\
Average temperature $\left({ }^{\circ} \mathrm{C}\right)$ & $28 \pm 4$ \\
Relative humidity $(\%)$ & 50
\end{tabular}

To produce electrospun PAN/SBA-15 webs a dispersion of $3 \mathrm{wt}$. \% of powder SBA-15 particles in DMF has been prepared by using a high shear homogenizer from IKA (France) with the speed of 18,000rpm during 30 minutes. The suspension has been then ultra-sonicated for 30 minutes at $50^{\circ} \mathrm{C}$. The calculated quantity, equivalent to $12 \mathrm{wt} \%$ of PAN polymer has been added into the prepared suspension of SBA15 in DMF and the solution has been stirred under moderate rotation at $70^{\circ} \mathrm{C}$ for 24 hours to prepare a homogeneous charged dispersion. The prepared solution has been electrospun immediately to avoid any phase separation.

The electrospinning of PAN/SBA-15: DMF solution has been performed by following the same electrospinning conditions of PAN:DMF solution that is mentioned in Table 1. Both described PAN:DMF and PAN-SBA-15:DMF solutions have been electrospun by means of a homemade, vertical electrospinning set up demonstrated in Figure 1.

This device consists of an automatic feed rate pump that feeds the polymer solution through the tube to the spinning needle with a predefined rate. The polymer solution comes out from a spinning needle located above the grounded Teflon ${ }^{\circledR}$ collector charged by the metallic particles. Between the needle and the collector, a high voltage electric field is applied by a high voltage direct current (DC) power, up to $30 \mathrm{kV}$. In electrospinning process the high electrostatic voltage charges the body of the polymer solution that leads to the surface deformation from a droplet into a conical shape, known as the Taylor cone. When the electrostatic force overcomes the surface tension of the polymer solution, a stable liquid jet is ejected from the cone's tip. Solvent evaporates while the jet is traveling through the air and ultrafine polymeric fibers are formed and collected on a grounded collector. ${ }^{29-32}$

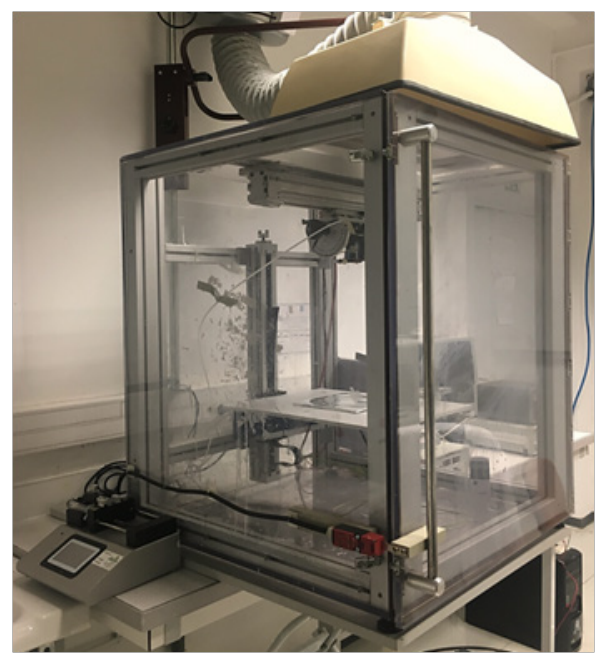

Figure I Laboratory scale electrospinning device.

The produced pristine and charged electrospun PAN webs have been observed for their structure, fibers morphology, and fibers diameter by using scanning electron microscope (SEM S-2360N Hitachi/Japan). The samples have been prepared and made conductive by gold coating before observation.

These samples have been observed with SEM to obtain images at different magnifications. The average diameter and deviation of the fibers within the scaffold have been calculated from 60 random measurements per SEM image via ImageJ software.

\section{Ibuprofen loading study on electrospun PAN nanofibers and electrospun PAN/SBA- I 5 webs}

The ibuprofen loading process has been performed on the pristine and charged electrospun PAN webs, for which the electrospinning conditions have been described previously. Ibuprofen loading procedure has been performed by immersing $400 \mathrm{mg}$ of electrospun webs into $300 \mathrm{mg}$ of ibuprofen in ethanol solution $(2 \mathrm{mg} / \mathrm{mL})$. Electrospun webs have been soaked into the prepared drug solution for 2 days under a continuous magnetic stirring at 100rpm at room temperature. In order to avoid any deformation of electrospun mats during the soaking procedure a Büchner funnel has been used. At pre-determined time intervals, during the loading procedure, aliquots of $3 \mathrm{ml}$ of the drug solution has been withdrawn and analyzed by UV-Vis PerkinElmer (Lambda 35) spectrophotometer to determine the concentration of the ibuprofen. The concentration changes of ibuprofen in ethanol at the start and end point of the loading procedure corresponds to the amount of the drug that has been absorbed into the electrospun webs samples. Once all UV-Vis absorbance measurements have been performed and no changes has been observed in ibuprofen absorbance, electrospun mats have been removed and washed with ethanol and then have been dried for the ibuprofen release experiment.

\section{Ibuprofen release study on loaded electrospun PAN/ SBA-I 5 webs in PBS solution}

The in vitro study of ibuprofen delivery from the drug loaded electrospun PAN/SBA-15 webs has been performed by immersing $23 \mathrm{mg}$ of the dried composite into $250 \mathrm{ml}$ of the phosphate buffered saline solution (PBS, $\mathrm{pH}$ : 7.4). The procedure has been carried out under constant stirring condition at $50 \mathrm{rpm}$ and at $37^{\circ} \mathrm{C}$ by using The Develop (RC-1) Dissolution Tester machine. The tester machine used for this experiment is illustrated in Figure 2. 


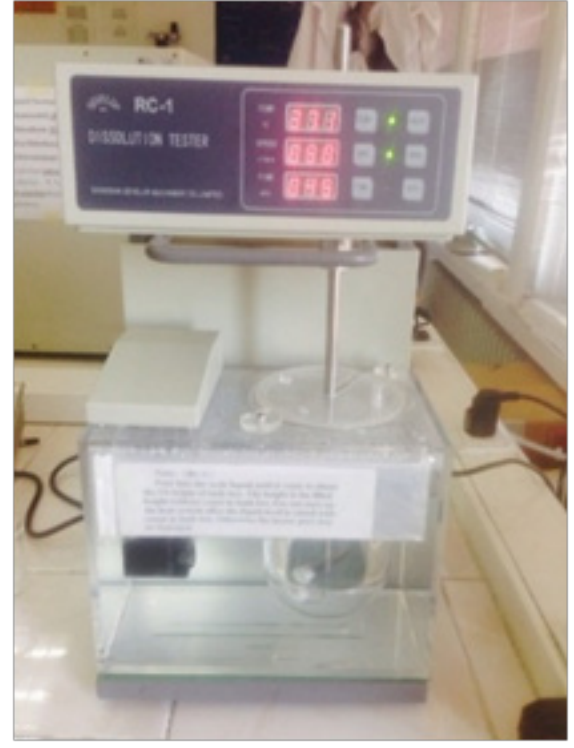

Figure 2 Develop (RC-I) Dissolution Tester machine, PAN/SBA-I5 in PBS solution under stirring.
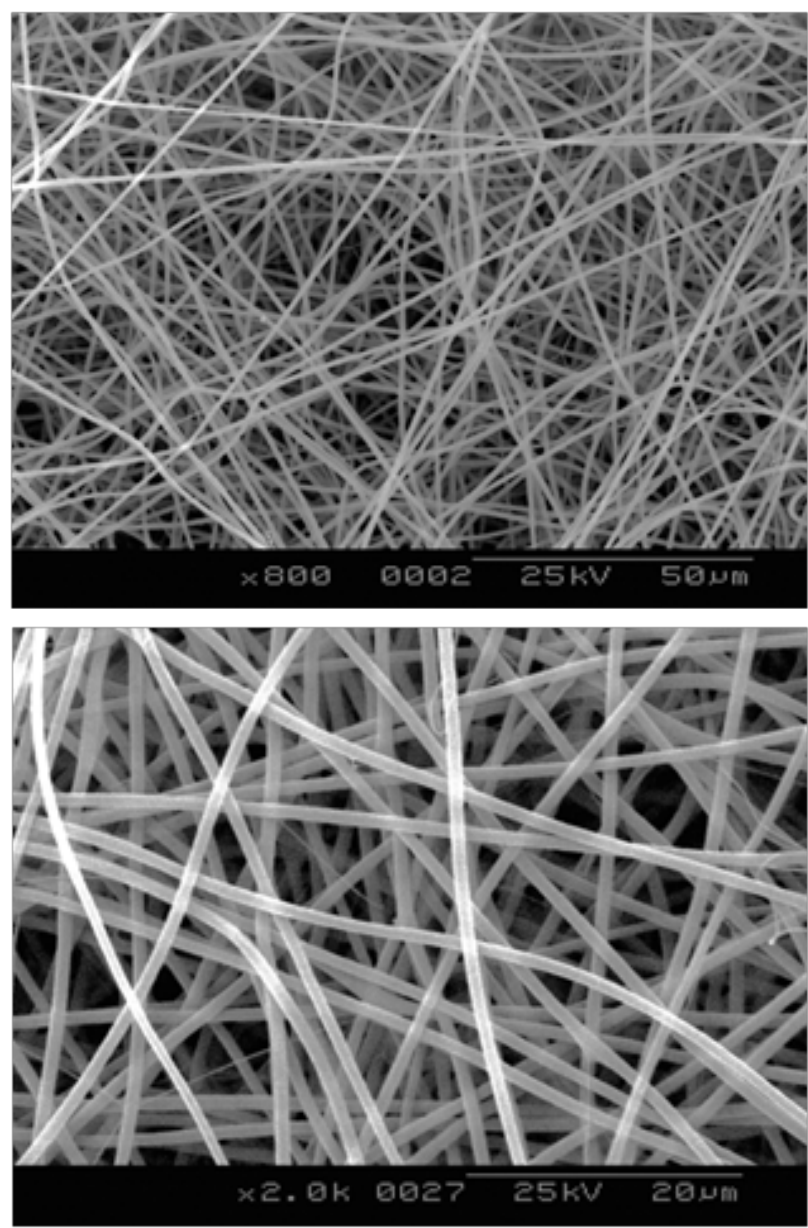

Figure 3 SEM micrographs of electrospun PAN nanofibers.

These SEM micrographs reveal a rough surface of nanofibers due to the presence of SBA-15 particles. It is observed that a large amount of charged particles are aligned along with the fiber axis. The results
Every 30 minutes, $5 \mathrm{ml}$ of the PBS solution has been withdrawn for UV-Vis spectroscopy measurement to evaluate the amount of ibuprofen delivered as a function of time in PBS solution.

\section{Results and discussion}

The morphological study of electrospun PAN nanowebs, demonstrates a good homogeneity and reproducibility for all samples obtained after different spinning times thanks to the stability of polymer solution jet. Fine and homogeneous nanofibers without any imperfections or solvent presence in the scaffold have been obtained. Figure 3 represents SEM micrographs of electrospun PAN nanofibers at different magnifications.

The random arrangement of nanofibers in nanoweb and porous structure of the electrospun membrane are observable on these photos. The SEM micrographs display the nanofibers with an average diameter of $972 \pm 24 \mathrm{~nm}$, measured by ImageJ software. Pristine PAN Electrospun nanoweb consists of sub-micron diameter fibers with the good uniformity of the diameter that confirms the optimal electrospinning conditions.

The SEM micrographs of produced electrospun PAN/SBA-15 webs with different magnifications are presented in Figure 4.
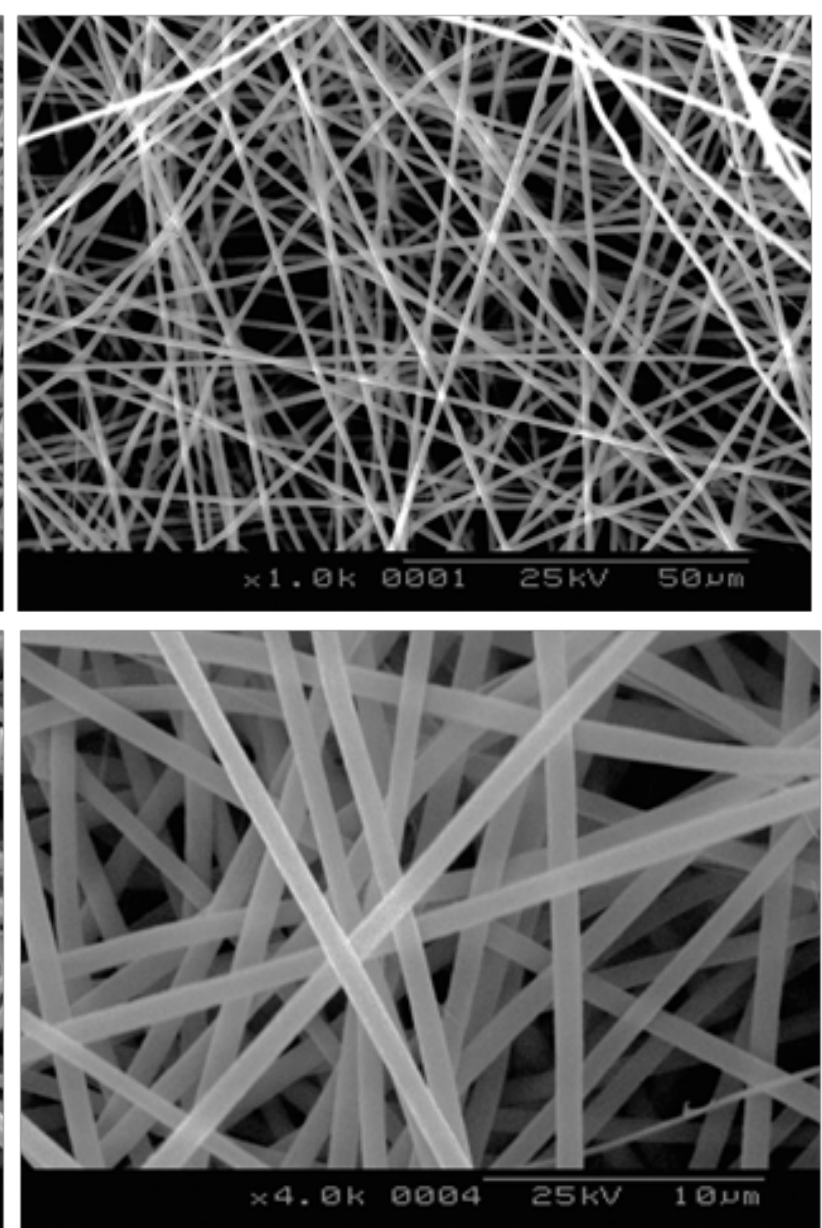

show that due to the aggregation of the SBA-15, the electrospun PAN/ SBA-15 web possesses coarser fiber diameter. 

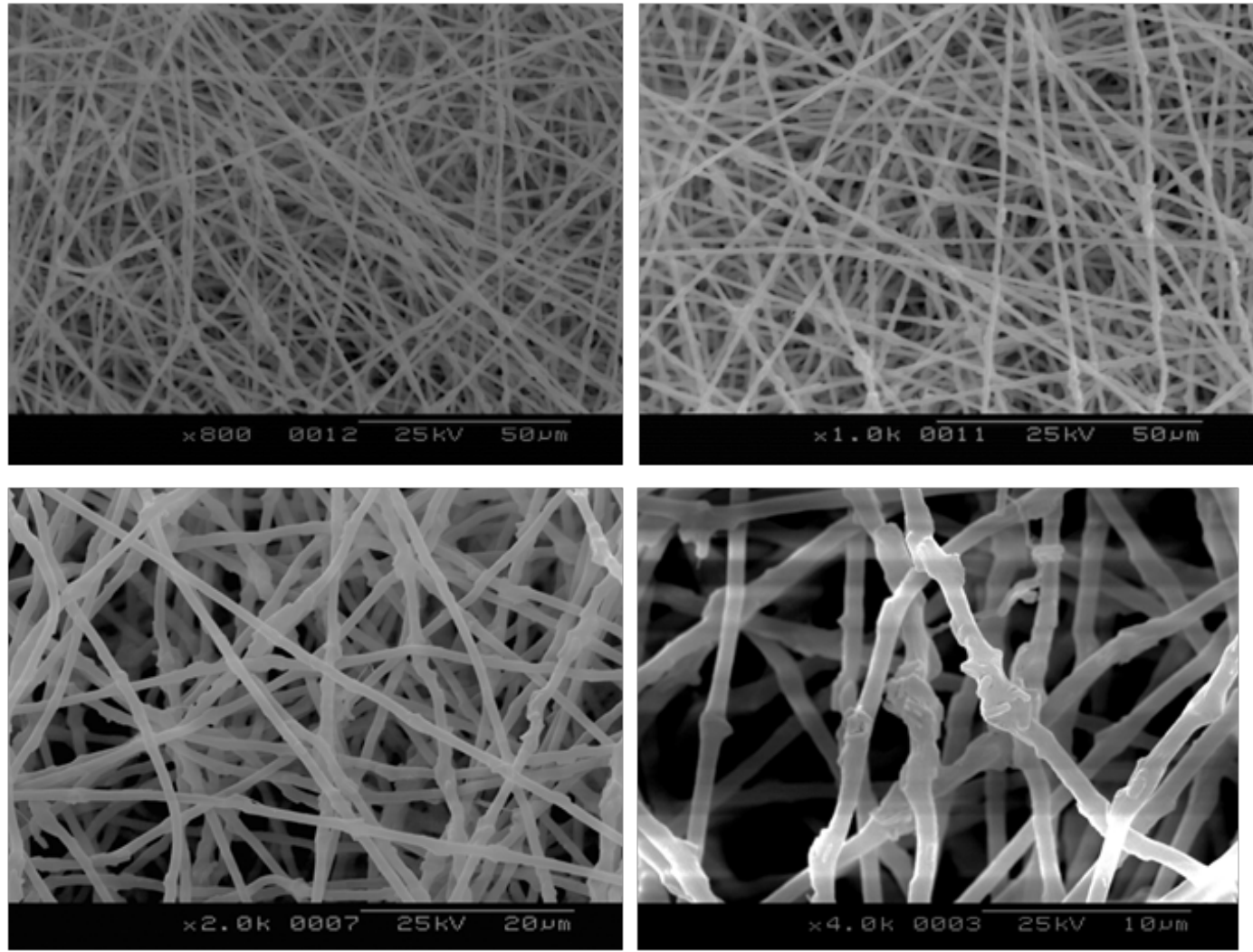

Figure 4 SEM micrographs of electrospun PAN nanofibers filled with SBA-I5 particles.

It should be considered that thermogravimetric measurement and $\mathrm{N}_{2}$ sorption isotherms characterization results in the recent study revealed that $78 \%$ of SBA- 15 particles are embedded in the electrospun webs and their mesoporosity is still accessible. ${ }^{1}$ These characteristics are evaluated in the context of drug delivery study.

UV-visible absorption measurements have been performed for electrospun PAN webs soaked in ibuprofen solution at $264 \mathrm{~nm}$. This selected wavelength corresponds to the maximum absorbance wavelength of ibuprofen in ethanol. The UV-vis results recorded at different interval times are demonstrated in Figure 5.

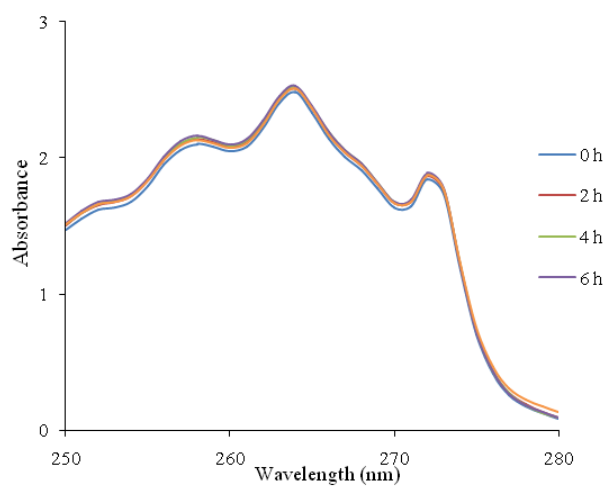

Figure 5 UV-Vis absorbance-wavelength curves of the ibuprofen ethanol solution - electrospun PAN nanofibers.

Figure 5 shows that after 24 hours, the absorbance of the ibuprofen loading solution containing of electrospun PAN nanofibers does not change by time increment. Hence, it can be concluded that electrospun PAN nanofibers are not capable to absorb ibuprofen.
On the contrary, in the case of electrospun PAN/SBA-15 webs soaked in drug solution different results have been obtained. The absorbance of the drug solution containing of electrospun PAN/SBA15 webs is measured at different interval times. The first and the last UV curves with 52 hours of interval time are shown in Figure 6.

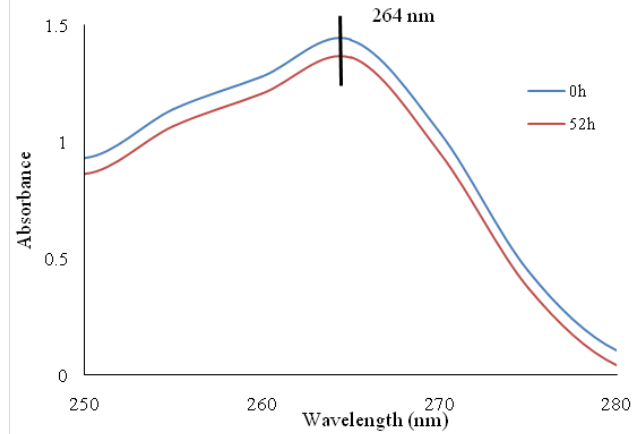

Figure 6 UV-Vis absorbance-wavelength curves of the first and the last UV/ Vis measurement - electrospun PAN/SBA-I5 composite in ibuprofen ethanol solution.

The ibuprofen concentration is calculated according to the BeerLambert law and the traced calibration curves at $264 \mathrm{~nm}$ wavelength. It can be clearly observed in Figure 6 that the concentration of ibuprofen in loading solution has been reduced with time increment at the wavelength of $264 \mathrm{~nm}$. This result confirms that the ibuprofen has been absorbed by SBA-15 particles embedded in electrospun PAN mats as according to the previous results electrospun PAN nanofibers do not absorb ibuprofen. The percentage of absorbed drug by SBA15 is calculated by division of concentration changes of ibuprofen in ethanol on its initial concentration as it is precised in equation 1. 


$$
\frac{\left(C_{I B U: \text { Ethanol }}(\text { initial })-C_{I B U: \text { Ethanol }}(\text { end })\right)}{C_{I B U: \text { Ethanol }}(\text { end })} \times 100
$$

\section{Equation 1 Absorbed drug calculation}

The obtained result indicates that $8.5 \mathrm{wt} . \%$ of ibuprofen has been absorbed by electrospun PAN/SBA-15 mats that corresponds to drug loading or $63.8 \mathrm{mg} / \mathrm{g}$ PAN/SBA-15 or $428 \mathrm{mg} / \mathrm{g}$ SBA- $15 .{ }^{17}$

After loading process, the drug release study has been performed on ibuprofen loaded electrospun PAN/SBA-15 mats in PBS solution. The effect of PBS on electrospun PAN mats has been investigated and the UV-vis results are reported in Figure 7. Figure 7 confirms that there is no effect of PBS on electrospun PAN/SBA-15 composite as no peak of absorbance is observable in the absorbance-wavelength curves.

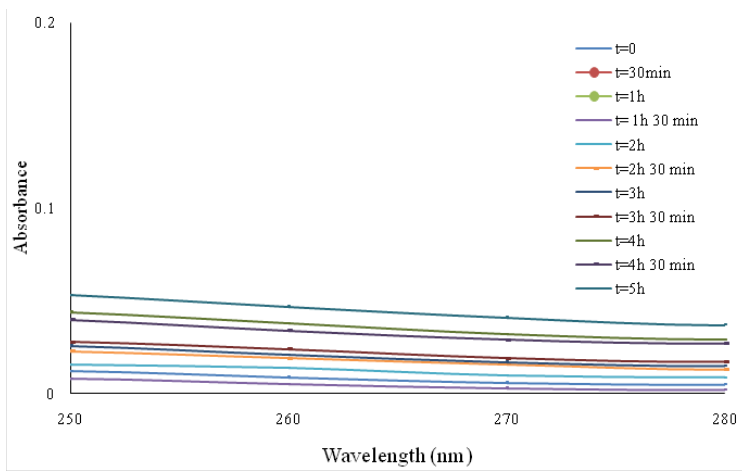

Figure 7 UV-Vis absorbance-wavelength curves of electrospun PAN/SBA-I5 mats in PBS solution.

The UV-Vis absorbance curves results of PBS solution containing IBU-loaded electrospun PAN/SBA-15 composite are reported in Figure 8 . The increase in intensity at about $220 \mathrm{~nm}$ with time increment in Figure 8 reveals the increase of ibuprofen concentration in PBS solution as a consequence of its release from the loaded electrospun mats filled with SBA-15 particles. The experiments were continued till no changes of ibuprofen absorbance in PBS solution have been observed. The trend of releasing profile of IBU-PAN/SBA-15 scaffold in PBS solution is shown in Figure 9.

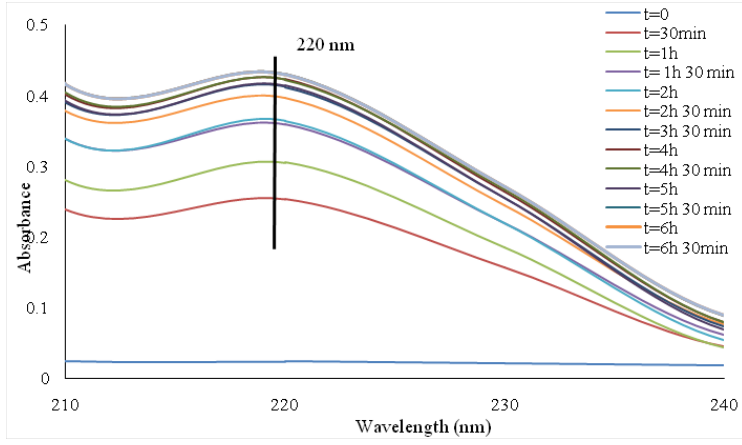

Figure 8 UV-vis absorbance-wavelength curves - electrospun IBU-PAN/SBA15 mats in PBS solution.

The trend of data presented in Figure 9 indicates that a burst release during the first hour has been occurred following by a slow release in the next hours to reach a plateau at $t>3 \mathrm{~h}$. Release profile is quite similar to those reported in the literature. ${ }^{33-35}$ The initial burst release can be due to ibuprofen molecules weakly entrapped on the surface or inside of the SBA-15 particles whereas the slow release can be due to the ibuprofen molecules that are strongly interacted with SBA-15 particles.

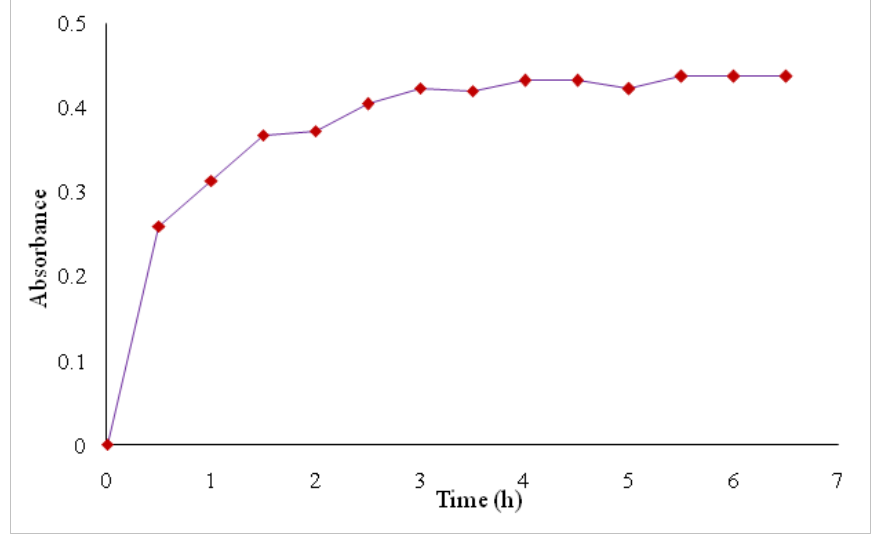

Figure 9 Releasing profile of IBU-PAN/SBA-15 mat in PBS solution.

\section{Conclusion}

Webs of pristine electrospun PAN nanofibers and the charged one with embedded SBA-15 ordered mesoporous silica particles have been produced via electrospinning technique in this study. Loading and releasing process of ibuprofen as model drug have been evaluated by UV-Vis spectroscopy measurement. It is concluded that electrospun PAN nanofibers do not absorb ibuprofen, whereas SBA15 particles increase the ability of drug absorption in electrospun PAN nanofibers charged with this kind of particles. It is found during release study that an initial burst release has been occurred at the very first hours followed by a very slow release pattern. The burst release can be because of the weak molecular interaction between ibuprofen and SBA-15 while the slow release is due to the hydrogen bond of the silanol group in the surface of SBA-15 particles with the carboxyl groups of ibuprofen.

\section{Acknowledgments}

Dr. Sliman Almuhamed is thanked for his preliminary study on Electrospinning of PAN nanofibers incorporating SBA-15-type ordered mesoporous silica particles

\section{Conflicts of interests}

Authors declare that there is no conflict of interest.

\section{References}

1. Almuhamed S, Khenoussi N, Bonne M, et al. Electrospinning of PAN nanofibers incorporating SBA-15-type ordered mesoporous silica particles. European Polymer Journal. 2014;54:71-78.

2. Zhao D, Feng J, Huo Q, et al. Triblock copolymer syntheses of mesoporous silica with periodic 50 to 300 angstrom pores. Science. 1998;279(5350):548-552.

3. Lee KY, Jeong L, Kang YO, et al. Electrospinning of polysaccharides for regenerative medicine. Advanced Drug Delivery Reviews. 2009;61(12):1020-1032.

4. Chakraborty S, Liao IC, Adler A, et al. Electrohydrodynamics: a facile technique to fabricate drug delivery systems. Adv Drug Deliv Rev. 2009;61(12):1043-1054.

5. Hekmati AH, Khenoussi N, Nouali H, et al. Effect of nanofiber diameter on water absorption properties and pore size of polyamide- 6 electrospun nanoweb. Textile Research Journal. 2014;84(19):2045-2055. 
6. Liu JJ, Wang CY, Wang JG, et al. Peripheral nerve regeneration using composite poly (lactic acid-caprolactone)/nerve growth factor conduits prepared by coaxial electrospinning. J Biomed Mater Res A. 2011;96(1):13-20.

7. Praveen Tyle. Controlled drug delivery: fundamentals and applications In: Robinson Joseph, Vincent HL Lee, editors. New York.: Marcel Dekker Inc; 1987.

8. Barbe C, Bartlett J, Kong L, et al. Silica particles: a novel drug-delivery system. Advanced materials. 2004;16(21):1959-1966.

9. Jin S, Ye K. Nanoparticle-Mediated Drug Delivery and Gene Therapy. Biotechnology progress. 2007;23(1):32-41.

10. Slowing II, Vivero-Escoto JL, Wu $\mathrm{CW}$, et al. Mesoporous silica nanoparticles as controlled release drug delivery and gene transfection carriers. Advanced drug delivery reviews. 2008;60(11):1278-1288.

11. John H Perrin. Sustained and controlled release drug delivery systems. In: Robinson Joseph R, editor. New York: Dekker; 1978.

12. Li Z, Su K, Cheng B, et al. Organically modified MCM-type material preparation and its usage in controlled amoxicillin delivery. $J$ Colloid Interface Sci. 2010;342(2):607-613.

13. Kresge CT, Leonowicz ME, Roth WJ, et al. Ordered mesoporous molecular sieves synthesized by a liquid-crystal template mechanism. Nature. 1992;359(6397):710-712.

14. Vallet-Regí M, Balas F, Arcos D. Mesoporous materials for drug delivery. Angew Chem Int Ed Engl. 2007;46(40):7548-7558.

15. Szegedi A, Popova M, Goshev I, et al. Effect of amine functionalization of spherical MCM-41 and SBA-15 on controlled drug release. Journal of solid state chemistry. 2011;184(5):1201-1207.

16. Gao L, Sun J, Zhang L, et al. Influence of different structured channels of mesoporous silicate on the controlled ibuprofen delivery. Materials Chemistry and Physics. 2012;135(2-3):786-797.

17. Izquierdo-Barba I, Sousa E, Doadrio JC, et al. Influence of mesoporous structure type on the controlled delivery of drugs: release of ibuprofen from MCM-48, SBA-15 and functionalized SBA-15. Journal of sol-gel science and technology. 2009;50(3):421-429.

18. Izquierdo-Barba I, Martinez A, Doadrio AL, et al. Release evaluation of drugs from ordered three-dimensional silica structures. European journal of pharmaceutical sciences. 2005;26(5):365-373.

19. Zeng W, Xue-Feng Q, Yan-Bo Z, et al. Organic modified mesoporous MCM-41 through solvothermal process as drug delivery system. Materials research bulletin. 2005;40(5):766-772.

20. Meynen V, Cool P, Vansant EF. Verified syntheses of mesoporous materials. Microporous and mesoporous materials. 2009;125(3):170 223.

21. García N, Benito E, Guzmán J, et al. Functionalization of SBA-15 by an acid-catalyzed approach: A surface characterization study. Microporous and Mesoporous Materials. 2007;106(1-3):129-139.
22. Zhao D, Feng J, Huo Q, et al. Triblock copolymer syntheses of mesoporous silica with periodic 50 to 300 angstrom pores. Science. 1998;279(5350):548-552.

23. Cavallaro G, Pierro P, Palumbo FS, et al. Drug delivery devices based on mesoporous silicate. Drug Deliv. 2004;11(1):41-46.

24. Xue JM, Tan CH, Lukito D. Biodegradable polymer-silica xerogel composite microspheres for controlled release of gentamicin. $J$ Biomed Mater Res B Appl Biomater. 2006;78(2):417-422.

25. Song SW, Hidajat K, Kawi S. Functionalized SBA-15 materials as carriers for controlled drug delivery: influence of surface properties on matrix-drug interactions. Langmuir. 2005;21(21):9568-9575.

26. Manzano Miguel, María Vallet-Regí. New developments in ordered mesoporous materials for drug delivery. Journal of Materials Chemistry. 2010; 20(27):5593-5604.

27. Colilla M, Manzano M, Vallet-Regí M. Recent advances in ceramic implants as drug delivery systems for biomedical applications. Int J Nanomedicine. 2008;3(4):403-414.

28. Hoffmann F, Cornelius M, Morell J, et al. Silica-based mesoporous organic-inorganic hybrid materials. Angew Chem Int Ed Engl. 2006;45(20):3216-3251.

29. Haghi AK, Akbari M. Trends in electrospinning of natural nanofibers. Physica status solidi. 2007;204(6):1830-1834.

30. Islam Shahidul, Rezaul Karim. Fabrication and characterization of poly (vinyl alcohol)/alginate blend nanofibers by electrospinning method. Colloids and Surfaces A: Physicochemical and Engineering Aspects. 2010;366(1):135-140.

31. Fang D, Liu Y, Jiang S, et al. Effect of intermolecular interaction on electrospinning of sodium alginate. Carbohydrate Polymers. 2011;85(1):276-279.

32. Sell SA, McClure MJ, Garg K, et al. Electrospinning of collagen/ biopolymers for regenerative medicine and cardiovascular tissue engineering. Adv Drug Deliv Rev. 2009;61(12):1007-1019.

33. Gonzalez Gema, Amaya Sagarzazu, Tamara Zoltan. Infuence of microstructure in drug release behavior of silica nanocapsules. Journal of drug delivery. 2013

34. Heikkilä T, Salonen J, Tuura J, et al. Evaluation of mesoporous TCPSi, MCM-41, SBA-15, and TUD-1 materials as API carriers for oral drug delivery. Drug Deliv. 2007;14(6):337-347.

35. Quevedo G Porras, Campero Celis A, Velasquez Ordonez C, et al. SBAtype mesoporous materials with cylindrical and spherical structures for the controlled loading and release of ibuprofen. Journal of Sol-Gel Science and Technology. 2018;85.(2):486-494. 